\title{
Biomedical applications of nanoscale tools and nano-bio interface: A blueprint of physical, chemical, and biochemical cues of cell mechanotransduction machinery
}

Rajiv Kumar*

NIET, National Institute of Medical Science, India.

Corresponding author: Rajiv Kumar, NIET, National Institute of Medical Science, India. Received date: April 20, 2021; Accepted date: June 14, 2021; Published date: June 17, 2021

Citation: R Kumar. (2021) Biomedical applications of nanoscale tools and nano-bio interface: A blueprint of physical, chemical, and biochemical cues of cell mechanotransduction machinery. Biomedical Research and Clinical Reviews. 4(2); DOI: 10.31579/2692-9406/064

Copyright:@ 2021 Rajiv Kumar, This is an open-access article distributed under the terms of the Creative Commons Attribution License, which permits unrestricted use, distribution, and reproduction in any medium, provided the original author and source are credited.

\section{Opinion}

A dream to have control over the cell behavior by nanoscale tools and nano-bio interface to mimic remodeling of cell mechanotransduction machinery, is an updated approach and the latest theme of current research.[1] To achieve such a goal, the nanofabrication technique plays a key role in designing novel nanoscale tools capable of stimulating the natural extracellular matrix (ECM). These nano-bio tools can create a valuable nanoscale interface, and finally, these advanced tools control cell behavior. Structurally and compositionally, the cells are too complicated and well equipped with remarkable features. It has a lot of complexity in it. The initial hurdle is the natural composition of cells and the surroundings of the nanoscale. The cell is too complicated, and it is a difficult and tough task to determine the features of its areas. The emergence of nanoscale tools, which are capable of analyzing and performing by applying single-molecule with high precision is helping for boosting cellular events for enhancing biomedical claims.[2] These tools and biomedical methods consist of nanomaterials that can perform as nanodevices, expose the cellular environment and simulate the cell-matrix interface. These biomedical methods are now considered major outfits for further analysis. [3] To detect the surface patterning of the cells and concerned topographies of cellular environments, these nanoscale devices, and 3D microporous scaffolds derived from nanomaterials are the main equipment applied to exploit the hidden areas and undiscovered activities of the cell components.

At the nanoscale, to achieve control of cellular functioning, there is a need to analyze the detail of the composition of cell compartments and the origin of interfaces using the aforementioned biomedical claims. If special emphasis will have applied to explore cell adhesion, signaling, and biochemical functionalization, then these discoveries can be fruitful and help understand the undiscovered features. How biomedical applications can control cell behavior, should be investigated. The fabricated biomimetic scaffolds and nanotools can be used to get a high temporal and spatial resolution of the cellular microenvironment to expose cell-cell, and cell-matrix, interfaces, and interactions for vascularization.[4] For successful drug delivery,[5-12] and to know how the fluid flow occurred, is important and the screening of biochemical routes is necessary. To determine these aspects, the use of the single-molecule resolution technique of high resolution and biomimetic scaffolds will be useful. A necessity is there to judge the specific features of engineered nano-bio interfaces to determine complicated hurdles, which are blocking the path of the discoveries as titled "biomedical applications of nanoscale tools and nano-bio interface: a blueprint cellular events". Herein, to accomplish this dream, the author is planning to design the blueprint of a suitable route to be implemented in the development of preventive and regenerated therapeutic, and that is looked for. The designed nanodevices and nanotools will emerge to originate smart interfaces for exploring the cellular mechanisms, and displayed the character of regenerative medicine, as the foremost goal and aspects of this discussion (Fig. 1). Therefore, the role of analysis and examination of nanotopographies contributions in exploring cell pathways, cellular dynamics, intracellular happenings, adherens junction, and mechanosensing is important in claiming new medical applications necessarily.[13] The routes of discovery are mandatory in exposing cell functioning and caring methodology, and quite helpful in designing new strategies required in controlling cell behavior. These nanotopographical machines (nanotools and nanodevices) can detect physical and chemical cues of the complex cellular events and mechanisms responsible for governing cell behavior, signaling pathways, and other complicated mechanisms happened within cells and tissues.[14] 


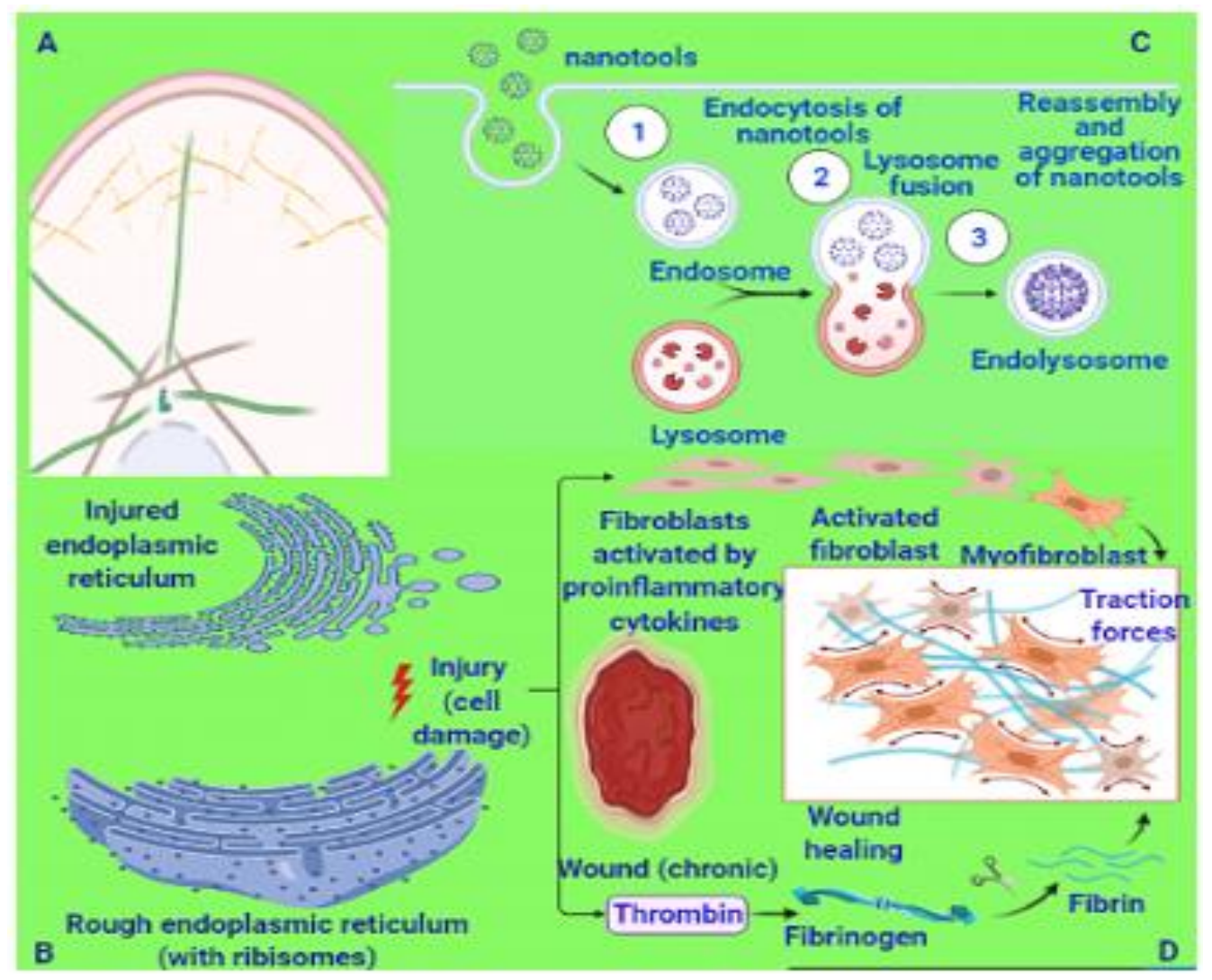

Figure 1: Schematic representation of the wound healing. Scaffold structural microenvironmental may be useful for tissue regeneration for bones. As scaffold pore structure (i.e., porosity and pore size), grain size, and surface topography, and (ii) how the mechanism scaffold microenvironmental cues guide bone tissue regeneration. " Adapted and created with permission from [biorender.com] and acknowledged.

The whole credit goes to nanotechnology for providing such wonderful tools and devices that are highly capable of finding out the stimuli-responsive interfaces responsible that govern and operate extracellular physical, chemical, and biochemical cues through intracellular sensing. The emergence of nanoengineered cell-nanomaterial interfaces, elucidation of signaling pathways, and the blueprint of cellular mechanotransduction machinery will play a key role in the innovations of new biomedical applications and will provide enormous opportunities looked for to fulfill the claims of biomedical science including accurate bioimaging, precise diagnostic, and novel nanotherapeutic. Now, it is assumed that these recent findings will become a reality to achieve biomedical claims. It has also been evidenced from the reported literature that nanotopographical machines can easily interact with cells and govern cell functioning and molecular happenings.[15] To determine the biological responses of the cell and tissue toward these nanodevices and nanotools, advanced investigations are required. These findings will be very helpful to do an accurate analysis and will find more proof to prove their potential in biomedical applications.

As mentioned earlier, the nanomachines and nanotools (nanoparticles, nanotopographies, mixed composite scaffolds) and originated nano-bio interfaces within cells are very effective as regenerative therapeutics and their impact as regenerative remedies will finally flag them as the best option in regenerative medical science. By applying these nanotools and nanodevices for detection, it will be easy to know the exact mechanism behind controlling the cellular behaviors and to find out proper pathways of cellular processes including adhesion, proliferation, migration, differentiation, morphology, hidden mechanisms, and molecular signaling pathways. These routes and pathways regulate cell behavior and signaling mechanism. Several possibilities will emerge there that will expose the perfect statistics of the chemical, and biochemical cues of the cell mechanotransduction machinery. These findings will play a crucial role in the development of a better drug delivery approach that can be utilized in the development of the regenerative therapeutic for cell regeneration and healing.[16] The interaction between cells and nanomaterials enhanced the frequency of active biological functionalities.[17] For the proof, the recently reported finding on the human neuroblastoma cell line, in which the gold surfaces having different levels of nanoroughness employed for regeneration is referred to here as a ready reference.[18] The sense of neurons actively responded toward these applied surfaces of nanotopography and enhanced regeneration. The development of scaffolding materials for recapitulating the cellular microenvironment and determining the physicochemical cues within cells is crucial in repairing damages of the cells/organs.[19] The use of gold nanoparticleintegrated scaffolds achieved the same result when it was applied to stem cell proliferation and differentiation. This phenomenon is quite helpful for the detection of a decline in cell adhesion at an earlier stage, and to determine its impact on the cell signaling pathways that can be influenced by nanostructured surfaces. It was also reported that the neurons lost polarity in the presence of nanoroughness surfaces during cell adherent processes.

The processes of necrosis and cell death are also directly linked with the roughness values of the nanostructures. Cell organelles and their mechanisms (Golgi apparatus fragmentation, and nuclear condensation) are not affecting too much by their presence and that is a good sign for the clinicians. It was also testified that the mechanisms of cytophilic or cytophobic behavior are directly governed by the surface topography of nanodevices and nanotools. Specific and functional adhesion of cells happened only onto flat gold stripes with a strong selfalignment of neurons that are portraying the route for the development of nano/bio-materials that are well equipped for generating precise nanostructuretriggered biological responses.[18] Intracellular and extracellular mechanisms of 
cellular proceedings decided various key mechanisms and pathways (single-cell, including proliferation, apoptosis, motility, differentiation, and mutations), and these nanotopographical designed nanotools and nanodevices offered new opportunities to expose the aforementioned features of various mechanisms up to a single-cell level (Fig. 2). These nanodevices and nanotools were used as microscopic imaging platforms for cell/tissue tracking in vitro and in vivo. The outcome of the analysis of the blueprint of mechanotransduction is hinting at the conversion ability of cellular mechanosensors to translate mechanical signals into biological signaling.[20] The processes in which mechanical vibrations are to be used to stimulate mechanotransduction and can be used further as a technique to control cell behavior.

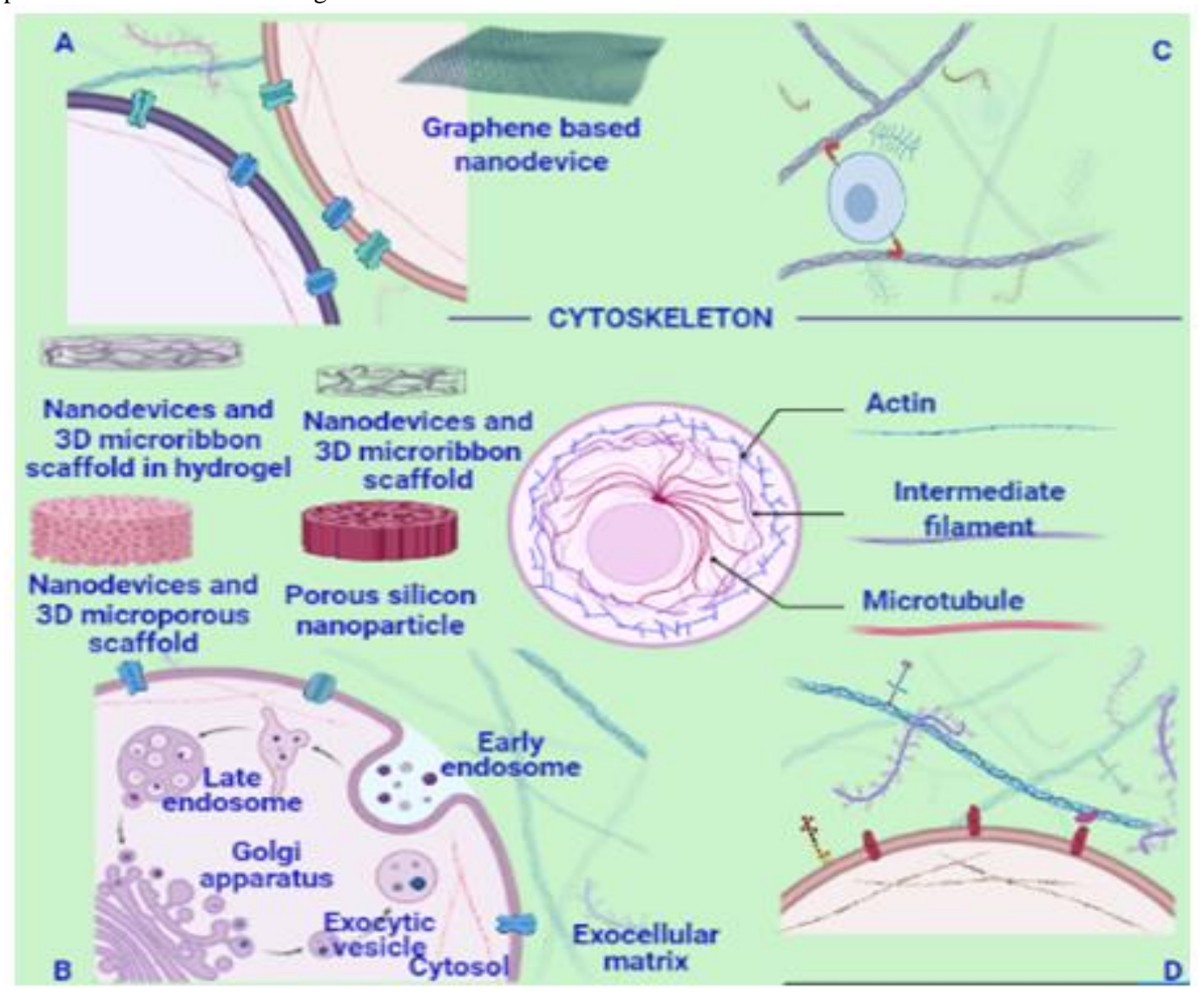

Figure 2: Schematic representation of the wound healing. Scaffold structural microenvironmental may be useful for tissue regeneration for bones. As scaffold pore structure (i.e., porosity and pore size), grain size, and surface topography, and (ii) how the mechanism scaffold microenvironmental cues guide bone tissue regeneration. . "Adapted and created with permission from [biorender.com] and acknowledged.

In these techniques, there is no chemical stimulation required, therefore, this could be a novel theme and will be very successful in drug discovery, clinical tissue engineering, and regenerative therapeutic and can be implemented. The nanoscale sinusoidal vibration technique will be a new strategy that can be utilized in the field of regeneration of cells and tissues. The technique of nanovibrational stimulation defines as 'nanokicking' processes, that are quite helpful in the aforementioned field.[21] Mechanotransductive mechanisms and kinase signaling pathways are crucial for the detection of cell differentiation efficiently and can be triggered in soft gel constructs. It was evident that the clinically relevant (three-dimensional) volumes of osteoblasts were used for bone grafting. An initial analysis noticed that the nanovibrational stimulation has no link or no role in the formation of biofilm formation at the time of bacterial existence.[22] These nano-bio types of machinery are very useful to investigate mechanotransduction in other fields of research having a biologically inert background. Further, these small tools are aligned with bioactive ligands for cellular functioning properly that will be independent of the mechanical properties.

The biocompatibility of nanoscale 3D scaffolds as tools depends on the compositions of components (collagen, fibrin, elastin, chitosan, silk fibroin, chitin, gelatin, and fibrinogen) used in the manufacturing efficiency and effectiveness that are propositionally interlinked with the physical parameters (stiffness, nanofibers, nanotubes, nanoparticles, structure, flexibility, pore size, and ligand density.[23] By enhancing the cellular environment in a physical, and chemical context, 3D scaffolds nanotools support the endothelial matrix during various cellular processes and mechanisms such as nutrient-gas transport, and cell health including cellular interactions, differentiation, and proliferation. The 3D scaffolds nano-compositions also assisted in drug delivery and execute activities similar to regenerative medicine.[24] The architectures of nanoscale 3D scaffold devices and the composition of cell-cell, cell-ECM interactions have many similarities and can easily cope with each other simultaneously. It happened because their sizes perfectly matched with it and fulfill the needs of each other in the range of nano. With wellequipped features, these nanodevices play key roles and emerged as deciding factors for the successful implementation of regenerative therapy.[25] It was highlighted earlier that the extracellular matrix consisted and configured with the same scale components and matched perfectly with the architecture of the small tools. Nanodevices are carrying nanomedicines for delivery and can make their impact on cell behavior and cell differentiation processes by the approach of the targeted drug delivery. Thus, the nanodevices are the constituents in the design and development of scaffolds and tissue engineering. Further, outlining the features of nanomaterials that can be utilized for controlling physical, 
chemical, and biochemical cues of cell mechanotransduction machinery will improve damaged cell functioning, tissue growth, and stop abnormal cell proliferation.

\section{Reference}

1. Hansel CS, Crowder SW, Cooper S, Gopal S, Joaio Pardelha Da Cruz M, De Oliveira Martins L, et al. (2019) NanoneedleMediated Stimulation of Cell Mechano transduction Machinery. ACS Nano.

2. Chen CS. (2008) Mechanotransduction - A field pulling together? J Cell Sci.

3. Gillespie PG, Müller U. (2009) Mechanotransduction by Hair Cells: Models, Molecules, and Mechanisms. Cell.

4. André EM, Passirani C, Seijo B, Sanchez A, Montero-Menei CN. (2016) Nano and microcarriers to improve stem cell behaviour for neuroregenerative medicine strategies: Application to Huntington's disease. Biomaterials.

5. Chhikara BS, Misra SK, Bhattacharya S. (2012) CNT loading into cationic cholesterol suspensions show improved DNA binding and serum stability and ability to internalize into cancer cells. Nanotechnology. 23(6).

6. Agarwal HK, Chhikara BS, Bhavaraju S, Mandal D, Doncel GF, Parang K. (2013) Emtricitabine prodrugs with improved anti-hiv activity and cellular uptake. Mol Pharm. 10(2): 467476.

7. Chhikara BS, Mandal D, Parang K. (2010) Synthesis and evaluation of fatty acyl ester derivatives of cytarabine as antileukemia agents. Eur J Med Chem. 45(10): 4601-4608.

8. Chhikara BS, Parang K. (2010) Development of cytarabine prodrugs and delivery systems for leukemia treatment. Expert Opin Drug Deliv. 7(12): 1399-1414.

9. Nasrolahi Shirazi A, Tiwari R, Chhikara BS, Mandal D, Parang K. (2013) Design and biological evaluation of cell-penetrating peptide-doxorubicin conjugates as prodrugs. Mol Pharm. 10(2): 488-499.

10. Chhikara BS, St. Jean N, Mandal D, Kumar A, Parang K. (2011) Fatty acyl amide derivatives of doxorubicin: Synthesis and in vitro anticancer activities. Eur J Med Chem. 46(6): 2037 2042.

11. Chhikara BS, Mandal D, Parang K. (2012) Synthesis, anticancer activities, and cellular uptake studies of lipophilic derivatives of doxorubicin succinate. J Med Chem. 55(4): 15001510 .

12. Agarwal HK, Chhikara BS, Quiterio M, Doncel GF, Parang K. (2012) Synthesis and anti-HIV activities of glutamate and peptide conjugates of nucleoside reverse transcriptase inhibitors. J Med Chem. 55(6): 2672-2687.
13. Ranella A, Barberoglou M, Bakogianni S, Fotakis C, Stratakis E. (2010) Tuning cell adhesion by controlling the roughness and wettability of 3D micro/nano silicon structures. Acta Biomater.

14. Mendes PM. (2013) Cellular nanotechnology: Making biological interfaces smarter. Chemical Society Reviews.

15. Chighizola M, Previdi A, Dini T, Piazzoni C, Lenardi C, Milani P, et al. (2020) Adhesion force spectroscopy with nanostructured colloidal probes reveals nanotopographydependent early mechanotransductive interactions at the cell membrane level. bioRxiv.

16. Mittal P, Singh S, Singh A, Singh IK. (2020) Current advances in drug delivery systems for treatment of Triple negative breast cancer (TNBC). Chem Biol Lett. 7(1): 1-12.

17. Yadid M, Feiner R, Dvir T. (2019) Gold NanoparticleIntegrated Scaffolds for Tissue Engineering and Regenerative Medicine. Nano Lett.

18. Brunetti V, Maiorano G, Rizzello L, Sorce B, Sabella S, Cingolani R, et al. (2010) Neurons sense nanoscale roughness with nanometer sensitivity. Proc Natl Acad Sci U S A.

19. Wang Y, Zhou F, Liu X, Yuan L, Li D, Wang Y, et al. (2013) Aptamer-modified micro/nanostructured surfaces: Efficient capture of ramos cells in serum environment. ACS Appl Mater Interfaces.

20. Vernerey FJ, Farsad M. (2011) A constrained mixture approach to mechano-sensing and force generation in contractile cells. J Mech Behav Biomed Mater.

21. Robertson SN, Campsie P, Childs PG, Madsen F, Donnelly H, Henriquez FL, et al. (2018) Control of cell behaviour through nanovibrational stimulation: Nanokicking. Philosophical Transactions of the Royal Society A: Mathematical, Physical and Engineering Sciences.

22. Hazrati H, Jahanbakhshi N, Rostamizadeh M. (2018) Fouling reduction in the membrane bioreactor using synthesized zeolite nano-adsorbents. J Memb Sci.

23. Kumar S, Singh D, Kumari P, Malik RS, Poonam, Parang K, et al. (2020) PEGylation and Cell-Penetrating Peptides: Glimpse into the Past and Prospects in the Future. Curr Top Med Chem. 20(5): 337-348

24. Piard CM, Chen Y, Fisher JP. (2015) Cell-Laden 3D Printed Scaffolds for Bone Tissue Engineering. Clinical Reviews in Bone and Mineral Metabolism.

25. Tsukioka T, Hiratsuka T, Nakamura M, Watanabe T, Kitamura Y, Isobe K, et al. (2019) An on-site preparable, novel bonegrafting complex consisting of human platelet-rich fibrin and porous particles made of a recombinant collagen-like protein. $\mathrm{J}$ Biomed Mater Res - Part B Appl Biomater.

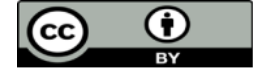

This work is licensed under Creative Commons Attribution 4.0 License
To Submit Your Article Click Here: Submit Manuscript

DOI: $10.31579 / 2692-9406 / 064$

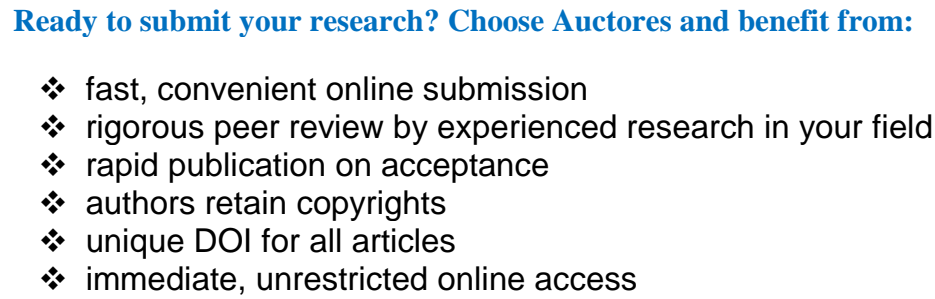

Ready to submit your research? Choose Auctores and benefit from:

* fast, convenient online submission

* rigorous peer review by experienced research in your field

* rapid publication on acceptance

* authors retain copyrights

* unique DOl for all articles

* immediate, unrestricted online access

At Auctores, research is always in progress.

Learn more www.auctoresonline.org/journals/biomedical-research-andclinical-reviews- 